\title{
Energetics of Defects and Diffusion Mechanisms in Graphite
}

\section{Citation}

Kaxiras, Efthimios and K.C. Pandey. 1988. Energetics of defects and diffusion mechanisms in graphite. Physical Review Letters 61(23): 2693-2696.

\section{Published Version}

http://dx.doi.org/10.1103/PhysRevLett.61.2693

\section{Permanent link}

http://nrs.harvard.edu/urn-3:HUL.InstRepos:10605459

\section{Terms of Use}

This article was downloaded from Harvard University's DASH repository, and is made available under the terms and conditions applicable to Other Posted Material, as set forth at http:// nrs.harvard.edu/urn-3:HUL.InstRepos:dash.current.terms-of-use\#LAA

\section{Share Your Story}

The Harvard community has made this article openly available.

Please share how this access benefits you. Submit a story.

Accessibility 


\title{
Energetics of Defects and Diffusion Mechanisms in Graphite
}

\author{
Efthimios Kaxiras and K. C. Pandey \\ IBM Research Division, T.J. Watson Research Center, Yorktown Heights, New York 10598 \\ (Received 10 June 1988)
}

\begin{abstract}
Extensive first-principles calculations are presented for the formation energies and migration barriers of elementary defects in graphite, as well as for the activation energy of atomic exchange. The calculations show that the long-held view that atomic diffusion in graphite occurs via the direct-exchange mechanism is incorrect, although more complicated exchange processes have not been ruled out. We find that diffusion in graphite is most likely mediated by vacancies with an activation energy which is lower than that of the interstitial and the direct-exchange mechanisms, and is in reasonable agreement with experiment.

PACS numbers: $71.55 . \mathrm{Ht}, 66.30 . \mathrm{Fq}$
\end{abstract}

The process of atomic diffusion, despite its technological importance, is still very poorly understood. Even the most basic question, what is the microscopic mechanism responsible for atomic diffusion, has not been answered in most cases of interest. Diffusion mechanics are generally divided in two broad categories: the exchange mechanism, in which atoms directly interchange positions and thereby migrate in the crystalline lattice, and the defect mechanism, in which the motion of a lattice defect (most commonly a vacancy or an interstitial) mediates the atomic migration. Naively, the exchange mechanism may appear to be advantageous since it does not depend on the creation of defects which is an energetically costly process. It has been found, however, that the activation energy of atomic exchange is in most cases very high. This is because during an exchange event large distortions are induced in the lattice. ${ }^{1,2}$ Defects on the other hand, once they have been created, can move through the lattice at a considerably smaller energy cost, and thus defect mechanisms are believed to dominate the diffusion process in most solids.

The single exception among covalent solids for which a non-defect mechanism is generally accepted to dominate diffusion is the case of graphite. ${ }^{3-7}$ This is based on comparison of diffusion data with theoretical calculations for graphite. ${ }^{3,8}$ However, these theoretical calculations are empirical and subject to numerous uncertainties. For example, different assumptions about the orbital configurations of a vacancy change the formation energy dramatically. ${ }^{8}$ Furthermore, a critical comparison of different diffusion mechanisms to experimental results did not lead to definite conclusions. ${ }^{7}$ Thus, in order to establish the dominant diffusion mechanism firstprinciples calculations are needed. This is particularly true since experimental information alone cannot identify the diffusion process. An unambiguous identification of the diffusion process would also require the calculation of dynamical factors (such as entropy and attempt frequency) which is beyond the scope of the present work. Here we shall concentrate on activation energies, which are well obtained by static zero-temperature calculations, and will prove adequate for comparing the various mechanisms that have been proposed in the literature. If atomic exchange turns out to be the dominant mechanism, graphite will represent an intriguing special case for the study of microscopic diffusion mechanisms which do not depend on the presence of defects. Recently, it was shown that an exchange mechanism (the concerted exchange) may be operative in Si (Ref. 9) although the exchange was not previously considered as a likely mechanism for the diamond lattice which has different bonding topology than graphite. The issue of non-defect diffusion mechanisms is of great theoretical and practical importance, since it has serious implications for the stability of doped materials and interfaces.

In this paper we report the first realistic, firstprinciples calculations for the energetics of the exchange mechanism and the defect mechanisms, vacancy and interstitial, in graphite. Given the extensive computational effort required for such calculations, we have limited the scope of our study to in-plane processes only. For the same reason, we have not investigated exchange mechanisms more complicated than the direct exchange, which might have low activation energy. The results of our calculations lead to conclusions contrary to the widely held view about atomic exchange in graphite. Namely, the direct-exchange mechanism has an activation energy higher than previously calculated $(10.4 \mathrm{eV}$ instead of 3.9 $\mathrm{eV}$, Ref. 3), and considerably larger than than experimental values (7-8 eV, Ref. 7). Moreover, the vacancy mechanism, which has an activation energy of at most $9.2 \mathrm{eV}$, is energetically preferred over the directexchange mechanism in graphite.

Our first-principles calculations employ normconserving pseudopotentials ${ }^{10}$ in the framework of localdensity-functional theory. ${ }^{11}$ This scheme allows for a fully self-consistent treatment of the electronic wave functions. The use of an appropriately large basis of plane waves and of a set of eight points in the Brillouin zone (which correspond to twelve points in the irreducible Brillouin zone of bulk graphite) to perform reciprocal-space averages gives realistic estimates for the energy cost involved in the various distortions, including the breaking of bonds. Convergence tests as a function 
of plane-wave cutoff were performed up to an energy of $32 \mathrm{Ry}$. In a typical example, we find that the activation energy of the direct-exchange mechanism changes by $0.06 \mathrm{eV}$ as the plane-wave cutoff is increased from 24 to $28 \mathrm{Ry}$. The large number of reciprocal-space sampling points and the large energy cutoff of the plane-wave basis, which are comparable to those used for highaccuracy bulk calculations in graphite, ${ }^{12}$ give a small error bar for the calculated energy differences. This error bar is estimated from the convergence studies to be less than $0.5 \mathrm{eV}$. The model on which the calculations were performed consists of a supercell geometry with eighteen atoms in each plane of graphite in the rhombohedral form (..ABCABC .. stacking). ${ }^{13}$ The reason for choosing the rhombohedral rather than the more common hexagonal form of graphite $(\ldots A B A B \ldots$ stacking $)^{13}$ is that the former possesses inversion symmetry which greatly facilitates the computations. We have verified that the results in hexagonal graphite, with defects in alternate planes, are the same as in rhombohedral graphite to better than $0.1 \mathrm{eV}$.

We shall consider first the direct-exchange mechanism on a plane of graphite, which consists of rotating two atoms by an angle $\theta$ around their bond center. During a complete exchange $\theta$ varies from $0^{\circ}$ to $180^{\circ}$. The ideal configuration $\left(\theta=0^{\circ}\right.$ or $\left.180^{\circ}\right)$ and the configuration corresponding to half-rotation $\left(\theta=90^{\circ}\right)$ are shown in Figs. 1 (a) and 1(b), respectively. In Fig. 2 we show the total energy of the system for $0^{\circ} \leq \theta \leq 180^{\circ}$, that is, for a complete in-plane direct exchange. From symmetry

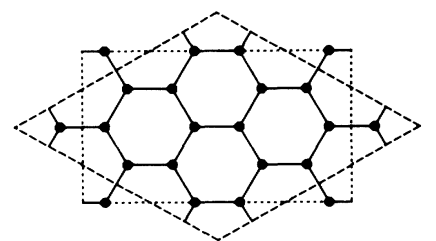

(a)

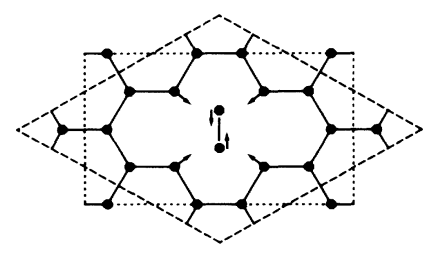

(b) (c)

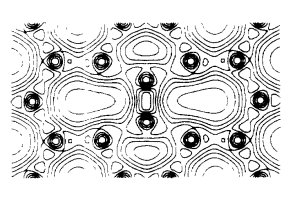

(d)

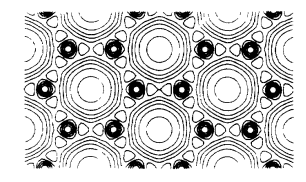

FIG. 1. (a) An ideal plane of graphite. The dashed lines outline the supercell used in the calculations. The dotted lines outline the area for which a valence-charge-density contour plot is shown in panel (c). (b) The $\theta=90^{\circ}$ configuration (half-rotation) of the direct-exchange path, which corresponds to the saddle point. Arrows indicate the direction of relaxation (not actual displacements). The dashed and dotted lines have the same meaning as in (a). (c) Contour plot of the valence charge density for the ideal configuration. The spacing of contours is 0.05 a.u. (d) Same as in (c) for the saddle-point configuration. considerations the $\theta=90^{\circ}$ configuration is an extremum of the total energy, but as seen from Fig. 2, it is a local minimum and not the maximum of the energy curve. The activation energy is determined by the highest of the energy maxima which must be crossed during the exchange process. Therefore, if this process is restricted to take place on the plane, the activation energy is not determined by the $\theta=90^{\circ}$ configuration, but rather by the $\theta=55^{\circ}$ or $125^{\circ}$ configuration, and is $13.7 \mathrm{eV}$.

The energy of the $\theta=55^{\circ}$ or $125^{\circ}$ configuration is high because at these in-plane configurations there is large bond compression. However, this compression can be relieved by out-of-plane motion of the exchanging atoms. The $\theta=90^{\circ}$ configuration on the other hand, involves bond stretching rather than bond compression, and its energy cannot be lowered by out-of-plane displacements. Instead, if the exchanging atoms are forced out of the plane at $\theta=90^{\circ}$, the energy increases. Thus, the energy of the $\theta=90^{\circ}$ configuration is a lower bound for the activation energy of the more general directexchange mechanism which includes out-of-plane displacements. In the following, since other mechanisms with lower activation energy will be encountered, it will suffice to identify the energy of the $\theta=90^{\circ}$ configuration as the minimum activation energy of the direct-exchange mechanism. This energy is $11.4 \mathrm{eV}$ before allowing for atomic relaxation (the exchanging atoms are at a relative distance equal to the ideal bond length of graphite, $1.42 \AA$ ).

We discuss next the effect of relaxation on the activation energy. On account of the extensive nature of the computations we have allowed only relaxation of the exchange-pair atoms relative to their bond center and symmetric relaxation of their four neighbors relative to the same reference point, as indicated in Fig. 1(b). The

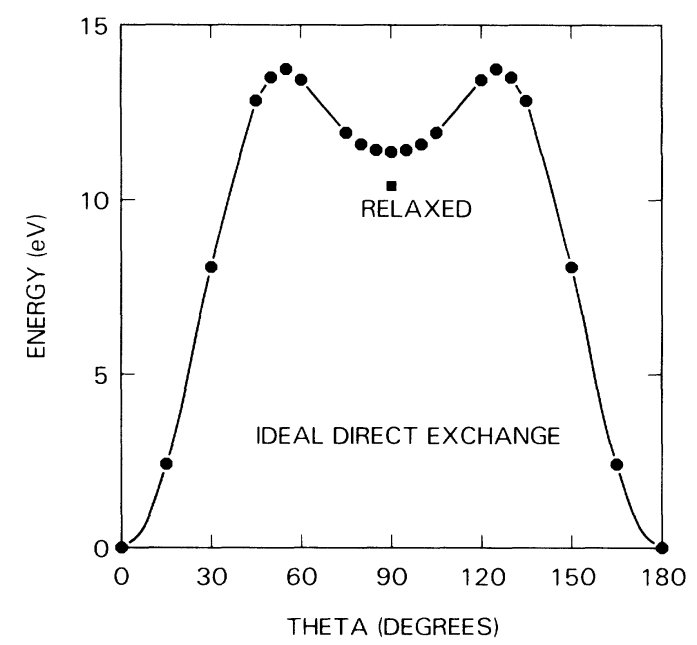

FIG. 2. The total energy vs rotation angle $\theta$ for the in-plane direct-exchange path. The dots are calculated energies; the line is a spline fit. The square gives the energy of the relaxed saddle-point configuration. 
relaxation of the exchange atoms relative to each other gives an energy gain of $0.8 \mathrm{eV}$ and the relaxation of their four nearest neighbors gives a gain of $0.2 \mathrm{eV}$. Thus the activation energy for the direct-exchange mechanism including relaxation is $10.4 \mathrm{eV}$. For comparison, empirical studies, including the relaxation of the exchange atoms relative to each other only, give an activation energy of $3.9 \mathrm{eV},{ }^{3}$ almost a factor of 3 too small.

The fully relaxed saddle-point configuration shows some interesting features of carbon bonding. At this configuration, the two atoms of the exchange pair move closer to each other, to a relative distance of $1.28 \AA$. This lies between a double and a triple bond in carbon, which have bond lengths 1.33 and $1.20 \AA$, respectively. ${ }^{14}$ The back bonds of the nearest neighbors are slightly stretched (one at 1.45 and the other at $1.43 \AA$ ), and the bond length between an exchange-pair atom and one of its neighbors is $1.50 \AA$. Thus, the two atoms of the exchange pair are effectively fourfold bonded, by a strong double bond to each other and by weaker single bonds to their neighbors. This bonding arrangement is illustrated in Fig. 1, where valence-charge-density contour plots for the ideal and the saddle-point configurations are compared in panels $1(\mathrm{c})$ and $1(\mathrm{~d})$. The bond between the exchange atoms at the saddle-point configuration [Fig. $1(\mathrm{~d})$ ] is different from other bonds: It lacks the doublehump feature of the normal graphite bonds and has considerably higher charge density. The weak bonds of the exchange atoms to their neighbors are also evident, containing a smaller amount of charge than normal bonds.

In the case of defect mechanisms, the activation energy is the sum of the formation energy and the migration barrier, that is, the energy to create a defect in the ideal lattice and the barrier to move it to a different site. We investigate first the interstitial mechanism. Since the present study is limited to in-plane diffusion only, the interplane interstitial, which might be important for atomic migration between planes, will not be considered in the following discussion. In the same vein, relaxations or migration paths which involve out-of-plane motion of an interstitial atom will not be considered here. This motion would lead to interplanar interstitial atoms which are known experimentally ${ }^{7}$ to diffuse rapidly at temperatures an order of magnitude lower $\left(200^{\circ} \mathrm{C}\right)$ than those required for in-plane diffusion $\left(2000^{\circ} \mathrm{C}\right)$. The only physically reasonable position for the in-plane interstitial atom is the center of an open hexagon.

We find that the formation energy of the ideal inplane interstitial is $19.5 \mathrm{eV}$. Empirical calculations also give a high formation energy for the interstitial, close to our result (18.1 eV, Ref. 3). The high formation energy leads us to conclude that the total activation energy of the in-plane interstitial will be higher than the activation energy of the direct exchange by many eV. This is due to the fact that the migration energy alone for the inplane interstitial should be roughly of the same magnitude $(10 \mathrm{eV})$ as the activation energy of the exchange, since both processes involve atomic interchanges. In fact the interchange in the interstitial mechanism takes place in a severely constrained environment because of the presence of the extra atom, and is probably a higherenergy process than the direct exchange. If one adds to the migration energy of the interstitial the formation energy (even lowered by a factor of 2 to $10 \mathrm{eV}$ as a result of relaxation, which is an extremely favorable and unlikely situation) the activation energy becomes at least $20 \mathrm{eV}$, making further study of the in-plane interstitial unnecessary.

We consider next the energetics of the vacancy mechanism. Our calculations give a formation energy of 7.8 $\mathrm{eV}$ for the ideal vacancy, which suggests the possibility of a low activation energy. To calculate the migration barrier, we need the energy of the saddle-point configuration relative to the equilibrium vacancy configuration. The vacancy in its equilibrium and saddle-point configurations is shown in Figs. 3(a) and 3(b), respectively. The energy of the unrelaxed saddle point is 1.9 $\mathrm{eV}$ with respect to the ideal equilibrium vacancy configuration, giving an activation energy of $7.8+1.9=9.7 \mathrm{eV}$. Even without considering atomic relaxation, our calculated value for the vacancy activation energy is lower than the activation energy of the fully relaxed exchange mechanism $(10.4 \mathrm{eV})$. This already casts doubt on the feasibility of the exchange mechanism in graphite. For comparison, the formation energy and the migration barrier of the vacancy as obtained by empirical calculations, without any relaxation, are 5.2 and $3.1 \mathrm{eV}$, respectively, giving an activation energy of $8.3 \mathrm{eV}$ (Ref. 3).

The atomic relaxation of the vacancy is complicated because of the possibility of symmetry-breaking JahnTeller deformations. An elaborate treatment of this possibility has been given by Coulson et al. ${ }^{8}$ in the context of empirical molecular-orbital theory. The formation en-

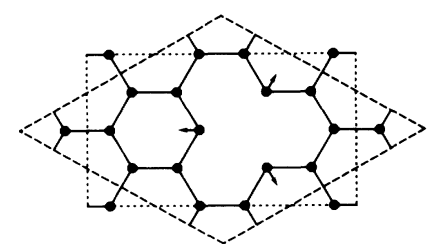

(a)

(b)

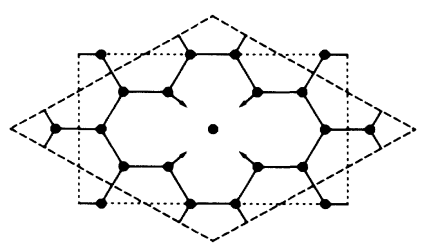

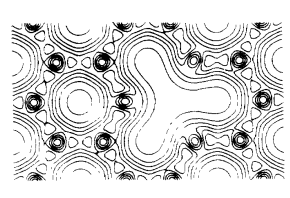

(c)

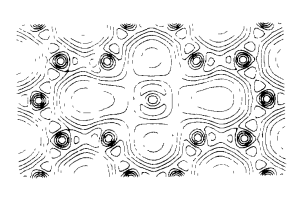

(d)
FIG. 3. (a),(c) The equilibrium vacancy configuration. (b),(d) The saddle-point vacancy configuration. Symbols are the same as in Fig. 1. 
ergy of the relaxed vacancy was estimated by these authors to be $10.7 \mathrm{eV}$, a factor of 2 larger than the empirical result of Dienes (5.2 eV, Ref. 3). Even the result of Ref. 8, however, is uncertain since a different orbital configuration could change the formation energy dramatically. Within our first-principles approach, because of technical reasons, we have been able to consider only the symmetric relaxation of the nearest neighbors of the vacancy for the equilibrium and the saddle-point configurations, as indicated in Figs. 3(a) and 3(b). In the equilibrium configuration, the three atoms adjacent to the vacancy site move radially away from it, thereby strengthening their back bonds. In the saddle-point configuration, the four nearest neighbors to the migrating atom move toward it, thus forming four weak bonds to it. These bonding arrangements are illustrated by valencecharge-density contour plots shown in Figs. 3(c) and $3(\mathrm{~d})$, for the equilibrium and the saddle-point configurations, respectively. The formation energy and migration barrier of the relaxed vacancy configurations are 7.6 and $1.6 \mathrm{eV}$. Thus, the activation energy of the vacancy including the relaxation described above is $7.6+1.6=9.2 \mathrm{eV}$, considerably lower than the relaxed direct-exchange mechanism $(10.4 \mathrm{eV})$ but still higher than the experimentally observed activation energy 7-8 eV (Ref. 7).

Our calculated activation energy for the vacancy is only an upper bound because we have not been able to consider more general relaxations, which require great computational effort as a result of the lower symmetry. The full relaxation of the vacancy may bring the activation energy closer to the experimental results and even lower than the direct-exchange activation energy. On the other hand, refinement of the relaxation for the direct-exchange mechanism is not expected to lower the energy significantly, since the first-neighbor relaxation energy $(0.2 \mathrm{eV})$ is already 4 times smaller than that of the exchange-pair atoms $(0.8 \mathrm{eV})$. Thus, the exchange mechanism considered above seems an unlikely process for diffusion in graphite, and the vacancy mechanism is energetically preferred. The possibility of a more complicated exchange path of lower energy cannot be ruled out, however. A search for such paths of low activation energy by means of the first-principles scheme employed in this work is not feasible at present.
In conclusion, we have shown that the most favorable in-plane diffusion mechanism in graphite is the vacancy mechanism with an upper bound for the activation energy of $9.2 \mathrm{eV}$. This value may become lower and in better agreement with experimental results of 7-8 eV by allowing further relaxations which are outside the computational scope of the present work. The direct-atomicexchange mechanism on the other hand has an activation energy considerably higher $(10.4 \mathrm{eV})$. These results are in sharp contrast to previous empirical calculations which estimated the activation energies of the directexchange and the vacancy mechanisms to be 3.9 and 8.3 $\mathrm{eV}$, respectively. ${ }^{3}$ It remains to be established whether a more complicated exchange path of lower activation energy exists, which can compete with the vacancy mechanism.

We wish to acknowledge useful discussions with J. D. Joannopoulos and thank N. D. Lang and E. Pytte for useful comments on the manuscript.

${ }^{1}$ H. B. Huntington and F. Seitz, Phys. Rev. 61, 315 (1942), and 76, 1728 (1949).

${ }^{2}$ C. Zener, Acta Crystallogr. 3, 346 (1950); F. Seitz, Acta Crystallogr. 3, 355 (1950).

${ }^{3}$ G. J. Dienes, J. Appl. Phys. 23, 1194 (1952).

${ }^{4}$ L. A. Girifalco, Atomic Migration in Crystals (Blaisdell, New York, 1964), pp. 113-116.

${ }^{5} \mathrm{~J}$. W. Corbett, Electron Radiation Damage in Semiconductors and Metals (Academic, New York, 1966), pp. 163-165.

${ }^{6}$ G. J. Dienes and D. O. Welch, Phys. Rev. Lett. 59, 843 (1987)

${ }^{7}$ M. A. Kanter, Phys. Rev. 107, 655 (1957).

${ }^{8}$ C. A. Coulson, M. A. Herraez, M. Leal, E. Santos, and S. Senet, Proc. Roy. Soc. London A 274, 461 (1963).

${ }^{9}$ K. C. Pandey, Phys. Rev. Lett. 57, 2287 (1986).

${ }^{10}$ G. B. Bachelet, H. S. Greenside, G. A. Baraff, and M. Schluter, Phys. Rev. B 24, 4745 (1981).

${ }^{11}$ P. Hohenberg and W. Kohn, Phys. Rev. 136, B864 (1964); W. Kohn and C. J. Sham, Phys. Rev. 140, A1133 (1965).

${ }^{12}$ M. T. Yin and M. L. Cohen, Phys. Rev. B 29, 6996 (1984).

${ }^{13}$ R. W. G. Wyckoff, Crystal Structures (Wiley, New York, 1973), Vol. 1, pp. 29 and 30.

${ }^{14} \mathrm{~L}$. Pauling, The Nature of the Chemical Bond (Cornell Univ. Press, Ithaca, 1944). 